\title{
Reoperaciones posadenoidectomía por hiperplasia adenoidea obstructiva
}

\section{Post-adenoidectomy reoperations due to obstructive adenoid hyperplasia}

\author{
Michelle Arroyo D¹, Mauricio Urrutia C1', Ariel Cisternas V1.
}

\begin{abstract}
RESUMEN
Introducción: La incidencia de reoperación posadenoidectomía, ya sea una segunda adenoidectomía o una amigdalectomía, no es conocida en nuestro medio. Publicaciones extranjeras muestran $2 \%$ de readenoidectomías y $8 \%$ de amigdalectomías posteriores.

Objetivo: Describir las adenoidectomías efectuadas en nuestro centro, evaluar la prevalencia de reoperaciones y buscar posibles factores asociados a éstas.

Material y método: Estudio retrospectivo descriptivo y analítico. Se revisaron fichas de pacientes adenoidectomizados por roncopatía con pausas respiratorias entre enero de 1999 y diciembre 2010. Se registraron datos demográficos, controles y nasofaringolaringoscopías (NFL). Se consignaron las reoperaciones (readenoidectomías y amigdalectomías).

Resultados: Se revisaron 106 fichas. Un 55,7\% de los pacientes eran hombres. A la NFL, $42 \%$ de los pacientes tenían adenoides grado 3 y $58 \%$ grado 4 de Parikh. Un 5,6\% de los pacientes fueron reoperados (1 adenoidectomía y 5 adenoamigdalectomías). Se observó diferencia significativa en edad $(p=0,04)$ y tamaño amigdalino $(p=0,004)$ entre los reoperados y lo no reoperados. No hubo asociación por sexo $(p=0,45)$, asma ( $p$ $=0,31)$ ni rinitis $(p=0,18)$. Sin embargo, a la regresión logística multivariada, ninguna variable se asoció significativamente de manera independiente con la necesidad de reoperación.
\end{abstract}

Conclusión: La prevalencia de reoperaciones fue similar a la publicada, no encontrándose asociación con otros factores.

Palabras clave: Adenoidectomía, reoperaciones, roncopatía, amigdalectomía.

\begin{abstract}
Introduction: The incidence of post-adenoidectomy reoperation, be it a second adenoidectomy or a tonsillectomy, is unknown within our environment. Foreign publications show a $2 \%$ of re-adenoidectomies and an $8 \%$ of ulterior tonsillectomies.
\end{abstract}

1 Médico. Servicio de Otorrinolaringología, Hospital San Juan de Dios. 
Aim: To describe the adenoidectomies performed at our center, to assess the prevalence of reoperations, and to seek possible associated factors to the latter.

Material y method: Descriptive and analytical retrospective assessment. A review was performed of records for patients that between January of 1999 and December of 2010 underwent adenoidectomy on account of snoring pathology. Demographics, controls, nasopharyngolaryngoscopies and reoperations (re-adenoidectomies and tonsillectomies) were recorded.

Results: The review entailed checking 106 records. 55,7\% of patients were men. $42 \%$ of patients had Parikh?s Grade III adenoids and 58\% showed Grade IV ones. 5,6\% of patients underwent reoperation. A significant difference could be observed in age $(p=0,04)$ and tonsillar size $(p=0,004)$ between those that had and had not undergone reoperation. There was no gender association $(p=0,45)$, neither for asthma $(p=0,31)$ or rhinitis $(p=0,18)$. Yet, by multivariate logistic regression, no variable was significantly associated by itself to the need for reoperation.

Conclusion: Reoperation prevalence was similar to that published, and no association to other factors was discovered.

Key words: Adenoidectomy, reoperatios, snoring pathology, tonsillectomy.

\section{INTRODUCCIÓN}

La adenoidectomía es la tercera cirugía pediátrica más comúnmente realizada en los EE.UU. ${ }^{1}$ y, sin lugar a dudas, también es una de las cirugías más efectuadas en la práctica otorrinolaringológica pediátrica a nivel nacional. Sus indicaciones más comunes son la otitis media aguda recurrente (OMAR), la disfunción tubaria, la rinosinusitis aguda recurrente (RSAR) y la crónica (RSC) y, la más frecuente, cubriendo $77 \%$ de las indicaciones, es la hiperplasia adenoídea asociada a roncopatía y síndrome de apnea e hipopnea obstructiva del sueño (SAHOS)2.

Cuando la indicación de la adenoidectomía es la hiperplasia adenoídea con roncopatía asociada a SAHOS, se realiza además una amigdalectomía si coexiste una hiperplasia amigdalina. No amigdalectomizar estaría justificado si al examen físico se evidencian amígdalas "pequeñas" (grado I y II de IV).

Los síntomas, tanto los ronquidos como las pausas respiratorias, suelen resolverse después de la adenoidectomía. Sin embargo, en algunas ocasiones éstos pueden persistir o recurrir por recrecimiento adenoíde $0^{3} 0$ al evidenciarse posteriormente a la cirugía una hiperplasia amigdalina ${ }^{4}$. En este sentido, la identificación y el entendimiento de los posibles factores propios del paciente asociados a riesgo de reoperación ayudaría a realizar un adecuado consentimiento informado $y$, eventualmente, a replantear la indicación de asociar una amigdalectomía en la primera intervención.

Sobre el riesgo de readenoidectomía, Dearking y cols. encontraron que los siguientes factores se aso- ciarían a esta reoperación: menor edad en la primera intervención quirúrgica, indicación de la adenoidectomía y enfermedad por RGE asociada. No estarían asociados la técnica quirúrgica, la experiencia del cirujano ni la alergia 0 asma asociada ${ }^{5}$.

Respecto a la amigdalectomía posadenoidectomía, un estudio de 2005 por Kay y cols. mostró que los pacientes más pequeños y aquéllos con amígdalas grandes tenían un riesgo mayor de requerir posteriormente una amigdalectomía 6 . Otro estudio más reciente, concluyó que este riesgo sería mayor en pacientes con indicación por obstrucción de vía aérea superior (versus OMAR, RSAR/RSC) y que dentro de este grupo el riesgo sería mayor en menores de 3 años, sexo femenino y amígdalas grandes 4 .

\section{OBJETIVO}

El objetivo del presente trabajo fue describir las adenoidectomías indicadas por hiperplasia efectuadas entre enero de 1999 y diciembre de 2010 en el Hospital San Juan de Dios, evaluar la prevalencia de reoperaciones (amigdalectomía y nueva adenoidectomía) y buscar posibles factores asociados a éstas.

\section{MATERIAL Y MÉTODO}

Estudio retrospectivo descriptivo y analítico. Se revisaron las fichas de pacientes adenoidectomizados 
por hiperplasia adenoídea obstructiva (HAO) entre enero de 1999 y diciembre de 2010. Se excluyeron las cirugías asociadas a punción aspirativa timpánica y/0 amigdalectomía. Se registraron datos demográficos, controles clínicos y nasofaringolaringoscopías (NFL), examen de elección en nuestro centro para la evaluación del tamaño adenoídeo, consignando grado de la hiperplasia adenoídea según la Clasificación de Parikh (Figura 1) ${ }^{7}$. Se registraron algunas comorbilidades y las reoperaciones, tanto las readenoidectomías como las amigdalectomías posteriores. Se analizaron los datos con el programa estadístico STATA13 usando prueba de $\mathrm{chi}^{2}$ y regresión de Cox para análisis multivariado.

\section{RESULTADOS}

Durante el periodo estudiado, se realizaron 188 adenoidectomías por HAO. Se pudieron evaluar 106 fichas $(56,4 \%)$.

Del total de pacientes, $56 \%$ fueron de sexo masculino. En la primera consulta, $52 \%$ pacientes tenían amígdalas grado I/IV y $48 \%$ grado II/IV (ninguno con mayor tamaño). En la evaluación previa a la cirugía, $7,5 \%$ de los niños tenían amígdalas grado III/IV. A la NFL, $39 \%$ de los pacientes tenían adenoides grado III de Parikh y $45 \%$ grado IV de Parikh, destacando que $16 \%$ de los pacientes no fue evaluado con NFL, sino sólo con radiografía de cavum. Otros hallazgos a la NFL, como signos de rinitis o septodesviación no fueron significativos.

La mediana de los tiempos de espera de la adenoidectomía fue de 18,2 meses (R.I. 10,1-25,8) desde la primera consulta y de 9,2 meses (2,6$17,3)$ desde la indicación de la cirugía. El 100\% de las adenoidectomías se efectuó por legrado con adenótomo de Barnhill. La edad promedio de los pacientes al operarse fue de 7,45 años (1 a 19 años).

Sólo 6 pacientes se reoperaron (5 adenoidectomías y 1 adenoamigdalectomía), equivalente al $5,7 \%$ de los casos. Al comparar el grupo de los reoperados con los no reoperados, se encontró una diferencia significativa en la edad de la primera

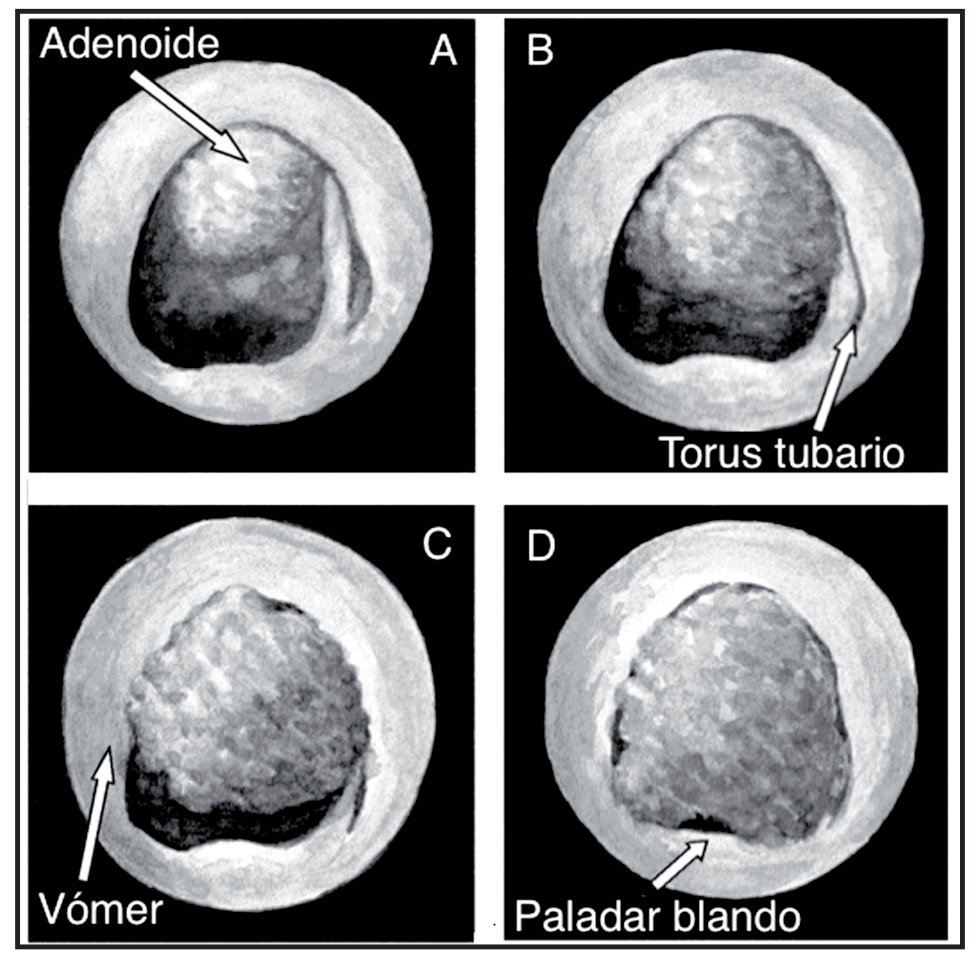

Figura 1. 
Tabla 1. Comparación de la características de los pacientes no reoperados y los reoperados

\begin{tabular}{|lccc|}
\hline Variable & $\begin{array}{c}\text { Pacientes no reoperados } \\
(\mathrm{n}=101)\end{array}$ & $\begin{array}{c}\text { Pacientes reoperados } \\
(\mathrm{n}=6)\end{array}$ & Significancia estadística \\
\hline Edad promedio de la primera cirugía & 7,6 años & 4,3 años & $\mathrm{p}=0,04$ \\
Sexo & $53,5 \%$ hombres & $66,7 \%$ hombres & $\mathrm{p}=0,45$ \\
Asma & $17,8 \%$ & 0 & $\mathrm{p}=0,31$ \\
Rinitis & $24,7 \%$ & $50 \%$ & $\mathrm{p}=0,18$ \\
Amígdalas hiperplásicas & 8 & 3 & $\mathrm{p}=0,004$ \\
\hline
\end{tabular}

cirugía: 4,3 años en los reoperados y 7,6 años en los demás $(p=0,04)$. Existió asociación con mayor tamaño de las amígdalas $(p=0,004)$. No hubo asociación por sexo $(p=0,45)$, asma $(p=0,31)$ ni rinitis $(p=0,18)$ (Tabla 1). Al hacer el análisis multivariado, se observó que ninguna variable se asoció significativamente de manera independiente con la necesidad de reoperación.

\section{DISCUSIÓN}

La patología hiperplásica adenoamigdalina, por su frecuencia e importancia sintomatológica, requiere un adecuado estudio y conocimiento de los factores que pueden influir en un mejor manejo del paciente. Es muy relevante indicar la cirugía adecuada para cada paciente, siendo muy importante el examen físico. En términos generales, en nuestro centro, estaríamos actuando bien, puesto que nuestra tasa de reoperación es similar a la descrita en la literatura: $5,6 \%$ (versus $2 \%-7,8 \%$ ) 4,6 .

Al analizar los distintos factores con un test de correlación multivariable, no se encontró ningún factor que influyera de manera independiente en la incidencia de reoperación. Es así, como no influyeron el sexo del paciente ni las comorbilidades registradas en la ficha. Sin embargo, la menor edad al momento de la primera cirugía y el mayor tamaño amigdalino al momento del ingreso para la cirugía fueron variables que mostraron cierta tendencia a influir en el riesgo de reoperación.

En consideración a este último punto, no es una sorpresa que pacientes con amígdalas hiperplásicas no resecadas requieran nuevas cirugías en el futuro si se mantienen sintomáticos, pero llaman la atención dos cosas. En primer lugar, que estos pacientes debieron haber sido sometidos a una adenoamigdalectomía en la primera intervención y no a una adenoidectomía exclusiva. Esto pudo haberse dado porque la indicación inicial fue sólo adenoidectomizar tras exámenes físicos sucesivos que no mostraron hiperplasia tonsilar $y / 0$ que en la evaluación intraoperatoria no se constató esta hiperplasia. Y en segundo lugar, que de los 3 pacientes que tenían amígdalas hiperplásicas y fueron reoperados, 2 se sometieron a una segunda adenoidectomía, con remisión total de los síntomas, pese a que en esta segunda intervención también debió hacerse una amigdalectomía. Esto podría estar explicado por un error del examen físico consignado al momento del ingreso del paciente para la cirugía y/o por haberse evidenciado que las amígdalas no eran hiperplásicas en la evaluación intraoperatoria.

Es así como el pequeño tamaño muestral, los errores asociados a la falta de concordancia interobservadores $\mathrm{y}$, en general, el incompleto registro de antecedentes, podrían estar influyendo en nuestros resultados. Sin embargo, es clara la tendencia (y además acorde a lo descrito internacionalmente) respecto a que la menor edad del paciente y el mayor tamaño tonsilar al momento de la adenoidectomía, impactarían sobre el riesgo de reoperación. Ahora bien, estudios posteriores e incluso prospectivos, podrían mostrarnos resultados más concluyentes.

\section{CONCLUSIONES}

Entre enero de 1999 y diciembre de 2010 se realizaron 188 adenoidectomías exclusivas por HAO, todas por legrado con adenótomo de Barnhill. Se observó $5,7 \%$ de reoperaciones, lo que mostró una tendencia a ser más frecuente en pacientes menores y con amígdalas más grandes. 


\section{BIBLIOGRAFÍA}

1. Cullen KA, Hall MJ, Golosinskiy A. Ambulatorysurgery in theUnitedStates, 2006. National Health Statistics Report 2009; 11:1-28. Disponible en: http://www. cdc.gov/nchs/data/nhsr/nhsr011.pdf. Consultado el: 08 de julio de 2012.

2. Erickson BK, Larson DR, St. Sauver JL, Meverden RA, ORVIDAS LJ. Changes in incidence and indications of tonsillectomy and adenotonsillectomy, 19702005. Otolaryngol Head Neck Surg 2009; 140(6): 894-901.

3. Buchinsky FJ, Lowry MA, IsAacson G. Do adenoids regrow after excision? Otolaryngol Head Neck Surg 2000; 23: 576-81.
4. Gov-Ari E, Mills JC, Basler KJ. Predictors of Tonsillectomy after Previous Adenoidectomy for Upper Airway Obstruction. Otolaryngol Head Neck Surg 2012; 146(4): 647-52.

5. Dearking AC, Lahr BD, Kuchena A, Orvidas LJ. Factors Associated with Revision Adenoidectomy. Otolaryngol Head Neck Surg 2012; 146(6): 984-90.

6. Kay DJ, Bryson PC, Casselbrant M. Rates and risk factors for subsequent tonsillectomy after prior adenoidectomy: a regression analysis. Arch Otolaryngol Head Neck Surg 2005; 131(3): 252-5.

7. Parikh SR, Coronel M, Lee J, Brown S. Validation of a new grading system for endoscopic examination of adenoid hypertrophy. Otolaryngol Head Neck Surg 2006; 135(5): 684-7. 